\title{
Shift-multiplexed holographic memory using the two-lambda method
}

\author{
George Barbastathis and Demetri Psaltis \\ Department of Electrical Engineering, Mail Stop 136-93, California Institute of Technology, Pasadena, California 91125
}

Received October 18, 1995

We present theoretical and experimental results on the application of the two-lambda method for prolonged readout of holographic memories to shift multiplexing implemented with a spherical-wave reference beam.

Key words: Shift multiplexing, two-lambda method, surface storage density. () 1996 Optical Society of America

Shift multiplexing ${ }^{1,2}$ is a method for holographic storage that uses nonplanar reference beams. A volume hologram recorded with a spherical-wave reference becomes Bragg mismatched when it is translated with respect to the readout beam. This effect can be used to multiplex holograms in the same recording medium. ${ }^{1,3}$ The required amount of translation $\delta$ between holograms is called shift selectivity and is typically of the order of a few to a few hundred micrometers, depending on the geometry, the material thickness, and the distance of the focus of the spherical wave to the hologram. Shift multiplexing is particularly convenient for holographic three-dimensional disks, ${ }^{4}$ because disk rotation naturally produces the required shift.

When photorefractive crystals are used to implement rewritable shift-multiplexed memories, an issue of concern is the undesired erasure of the recorded holograms during readout. Techniques have been developed for fixing holograms thermally ${ }^{5}$ or electrically ${ }^{6,7}$ Alternatively, holograms can be sustained by periodic refreshing. 8 Here we consider the twolambda method, in which the recording and readout wavelengths are different. ${ }^{9}$ The allowable readout time is prolonged if the readout wavelength is selected such that the absorption is lower and hence erasure is reduced. Because the Bragg matching condition at the new wavelength is modified, only a portion of the angular spectrum of the recorded holograms can be Bragg matched with a single plane wave. It is possible, however, to reconstruct in its entirety a single hologram recorded with a plane wave by reading out the hologram with a spherical beam. ${ }^{10}$ In this Letter we show how the two-lambda technique applies to shift-multiplexed memories, in which holograms are recorded and read out with spherical-wave references.

We use the subscript 1 for quantities associated with the recording wavelength $\lambda_{1}$ and the subscript 2 for the readout wavelength $\lambda_{2}$. The geometry is shown in Fig. 1. The holographic material occupies the region $-\infty<x, y<+\infty, \quad|z|<L / 2$. The recording reference beam (at wavelength $\lambda_{1}$ ) is a spherical wave originating from $\left(0,0,-z_{1}\right)$. For now, we consider the signal beam to be a single plane-wave component propagating at angle $u_{1} \approx \sin u_{1}$ (paraxial approximation) with respect to the hologram normal. The readout spherical wave (at wavelength $\left.\lambda_{2}\right)$ originates at $\left(\delta, 0,-z_{2}\right)$. Un- der the Born, paraxial, and constant modulation depth approximations $\mathrm{s}^{2,11}$ the diffracted field can be calculated analytically. The reconstruction is a plane wave if

$$
\lambda_{1} z_{1}=\lambda_{2} z_{2} .
$$

This change in focal distance compensates for the curvature mismatch between the recording and readout reference wave fronts. The plane wave hologram is Bragg matched at the readout wavelength if it is translated relative to the readout reference by

$$
\delta_{B}=\frac{1}{2}\left(\frac{\lambda_{2}}{\lambda_{1}}-1\right) u_{1} z_{2} .
$$

If Eqs. (1) and (2) are both satisfied, the reconstruction is a plane wave propagating at angle $u_{2}$ satisfying (in the paraxial approximation) the condition

$$
u_{2} / u_{1}=\lambda_{2} / \lambda_{1} .
$$

Once the hologram is Bragg matched after shifting by $\delta_{B}$, an additional translation by the shift Bragg selectivity

$$
\delta_{2}=\frac{\lambda_{2} z_{2}}{u_{2} L}=\frac{\lambda_{1}}{\lambda_{2}} \delta_{1}
$$

will make the reconstruction go away. A separate plane-wave hologram recorded at that shifted position

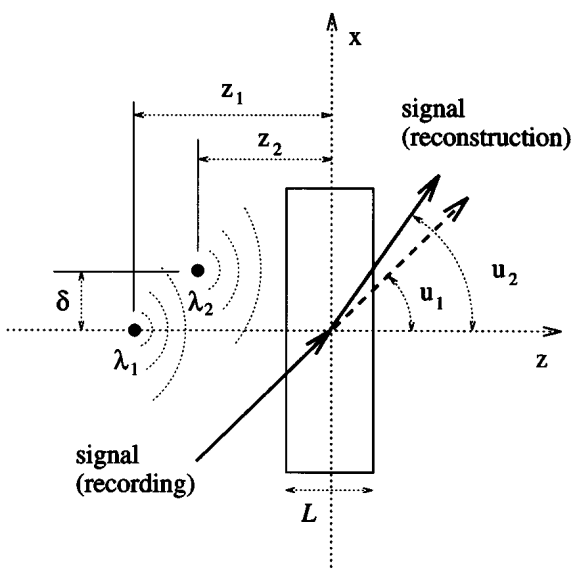

Fig. 1. Geometry for the two-lambda technique with shift multiplexing. 
Table 1. Two-Lambda Equations Including Refraction and Dispersion

\begin{tabular}{ll}
\hline Bragg selectivity & $\delta_{1}=\frac{\lambda_{1}^{\prime}\left[n_{1} z_{1}^{\prime}-\left(n_{1}-1\right) L / 2\right]}{u_{1}^{\prime} L}+\frac{\lambda_{1}^{\prime}}{2(\mathrm{NA})_{1}}$ \\
& $\delta_{2}=\frac{\lambda_{2}^{\prime}\left[n_{2} z_{2}^{\prime}-\left(n_{2}-1\right) L / 2\right]}{u_{2}^{\prime} L}+\frac{\lambda_{2}^{\prime}}{2(\mathrm{NA})_{2}}$ \\
Focusing condition & $\lambda_{1}^{\prime}\left[z_{1}^{\prime}-\left(1-\frac{1}{n_{1}}\right) \frac{L}{2}\right]=\lambda_{2}^{\prime}\left[z_{2}^{\prime}-\left(1-\frac{1}{n_{2}}\right) \frac{L}{2}\right]$ \\
Bragg matching condition & $\delta_{B}=\frac{1}{2}\left(\frac{n_{1} \lambda_{2}^{\prime}}{n_{2} \lambda_{1}}-1\right) \frac{u_{1}^{\prime}}{n_{1}}\left[n_{2} z_{2}^{\prime}-\left(n_{2}-1\right) L / 2\right]+\frac{\lambda_{2}^{\prime}}{2(\mathrm{NA})_{2}}$ \\
Reconstructed band & $\Delta u_{2}=\frac{\lambda_{1}^{\prime}}{\left(\frac{n_{1} \lambda_{2}^{\prime}}{n_{2} \lambda_{1}^{\prime}}-1\right) u_{1 c}^{\prime} L}$ \\
\hline
\end{tabular}

can be observed without interference. In Eq. (4), $\delta_{1}=$ $\lambda_{1} z_{1} /\left(u_{1} L\right)$ is the shift selectivity at the recording wavelength. ${ }^{2}$ Note that if $\lambda_{2}>\lambda_{1}$, then $\delta_{2}$ is smaller than $\delta_{1}$. Because holograms are recorded $\delta_{2}$ apart, packing is denser than for the case in which readout is intended to be at wavelength $\lambda_{1}$.

In the analysis so far we have neglected refraction and dispersion effects and the finite numerical apertures $(\mathrm{NA})_{1}$ and $(\mathrm{NA})_{2}$ of the spherical waves. The properly modified formulas are given in Table 1, where the primed quantities are measured in air and $n_{1}$ and $n_{2}$ are the refractive indices. In general, $(\mathrm{NA})_{2}<$ $(\mathrm{NA})_{1}$ because the required shift $\delta_{B}$ introduces edge effects.

We verified these theoretical predictions experimentally, using a thin Fe-doped $\mathrm{LiNbO}_{3}$ crystal grown by Deltronics. The experimental parameters were $\lambda_{1}^{\prime}=488 \mathrm{~nm}, \lambda_{2}^{\prime}=632.8 \mathrm{~nm}$, $u_{1}^{\prime} \approx 40^{\circ}=0.698 \mathrm{rad}, n_{1}=2.3533, n_{2}=2.2935$, $L=250 \mu \mathrm{m}$, and $(\mathrm{NA})_{1} \approx 0.1$. The quoted values of refractive index were calculated by interpolation of the data given in Ref. 12. This experiment was done with a plane-wave signal beam. The measured values of $\delta_{B}, \delta_{1}$, and $\delta_{2}$ are plotted in Fig. 2 together with the theoretical predictions derived from the formulas of Table 1. $(\mathrm{NA})_{2}$ was estimated to be 0.014 . We made the measurements for the $\lambda_{2}$ readout by first refocusing the readout beam according to the theoretical prediction for $z_{2}^{\prime}$.

An information-bearing signal occupies a finite angular bandwidth around the carrier $u_{1 c}$. Because $\delta_{B}$ [Eq. (2)] is different for each spatial frequency component, the entire hologram cannot be Bragg matched at the same time. The reconstruction consists of the portion of the angular spectrum lying in the range

$$
\Delta u_{2}= \pm \frac{2 \lambda_{1}}{\left[\left(\lambda_{2} / \lambda_{1}\right)-1\right] u_{1 c} L} .
$$

For Fourier-transform holograms, Eq. (5) implies that a slice of size $\Delta x=2 \Delta u_{2} F$ (where $F$ is the focal length of the lens used for the Fourier transformation) is reconstructed. For image-plane holograms the re- construction is low-pass filtered with a cutoff $\Delta w=$ $\Delta u_{2} / \lambda_{2}$. The modified version of Eq. (5) accounting for dispersion is given in Table 1.

We used the same experimental setup to record a single Fourier-plane hologram (focal length $F=20 \mathrm{~cm}$ ) of a transparency with $z_{1}^{\prime}=22.5 \mathrm{~mm}$. The reconstruction at wavelength $\lambda_{1}$ is shown in Fig. 3(a). Two reconstructions at $\lambda_{2}$ (obtained by changing $\delta_{B}$ ) are shown in Figs. 3(b) and 3(c). As predicted, only a
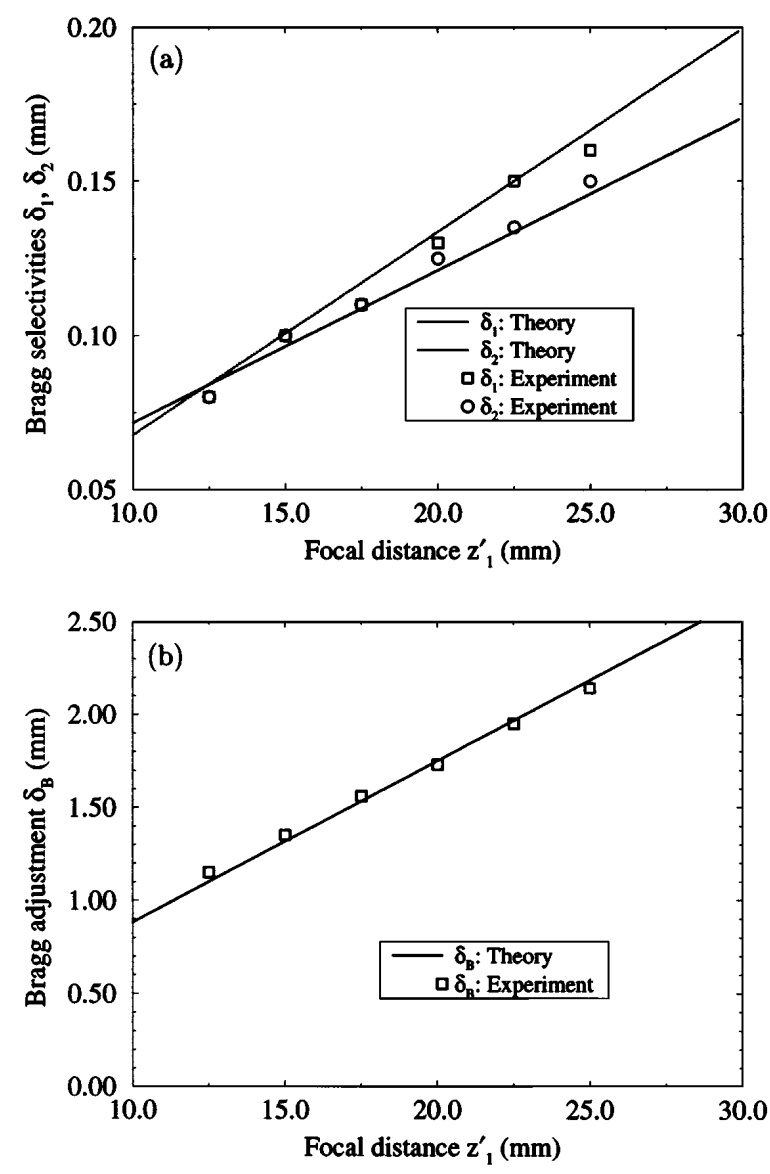

Fig. 2. Experimental results for the Bragg matching and selectivity properties of the two-lambda method applied to shift-multiplexed holograms. 


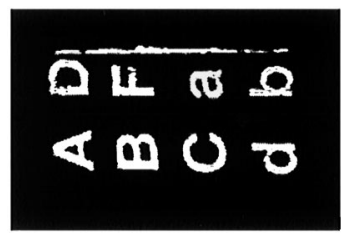

(a)

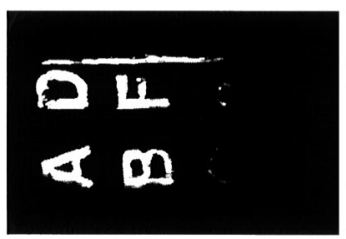

(b)

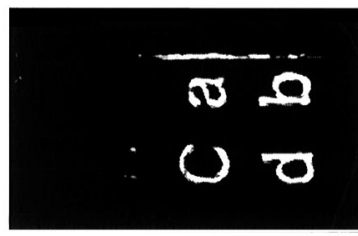

(c)

Slice \#1 Slice \#2

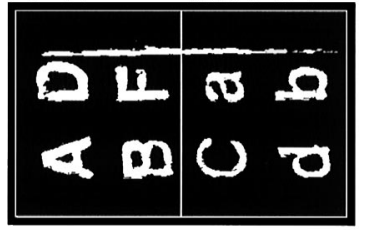

(d)
Fig. 3. Hologram reconstructions obtained with the twolambda method.

slice of the stored image is obtained at one time. The width of the slice is $0.7 \mathrm{~mm}$, in agreement with Eq. (5). If we continuously shift the medium, a sliding window of the stored image will appear on the CCD. In addition, the reconstruction as viewed through this sliding window is also shifting because of the motion of the medium. A time-delay-and-integrate detector array can compensate for this motion and integrate the response to produce a complete, unblurred image. Such a detector was simulated in software to produce the complete reconstruction shown in Fig. 3(d). Alternatively, we can set the size of the recorded images in the $x$ direction equal to the width of the reconstructed slice. Either method results in the complete reconstruction of the stored images at the expense of reduced storage density.

The surface storage density of a shift-multiplexed memory can be expressed as

$$
\mathcal{D}=\left(N_{p x} N_{p y}\right) /\left(s_{1} \delta_{2}\right),
$$

where $N_{p x}$ and $N_{p y}$ are the number of spatial light modulator pixels in the $x$ and $y$ directions, respectively, and $s_{1}$ is the transverse size of the signal beam. For Fourier-plane recording we can relate $N_{p x}$ to the geometry of the holographic system by imposing the requirement that the angular bandwidth of the stored holograms (and hence the hologram thickness) be matched to the width of the allowable reconstructed angles (the size of the spatial light modulator). Substituting Eqs. (5) and (6), we obtain

$$
\mathcal{D}=\frac{2 N_{p y}}{\left(\frac{\lambda_{2}}{\lambda_{1}}-1\right) \lambda_{1} z_{1}} .
$$

Equation (7) is valid only if the spatial light modulator's size is large enough to accommodate the entire strip. Notice that when $\lambda_{2}=\lambda_{1}$ the strip is infinite, and therefore Eq. (7) does not hold. As an example, if $N_{p y}=1000, z_{1}^{\prime}=1.3 \mathrm{~mm}, \lambda_{1}^{\prime}=488 \mathrm{~nm}$, and $\lambda_{2}^{\prime}=633 \mathrm{~nm}$, then $\mathcal{D}=10.6 \mathrm{bits} / \mu \mathrm{m}^{2}$. A similar derivation can be carried out for the density of the image-plane geometry, and it leads to the same expression as Eq. (7).

We have presented the theory of the two-lambda technique applied to spherical reference wave holograms and verified the results experimentally. Shift multiplexing is also achieved with a one- or twodimensional fan of plane waves (array method ${ }^{1}$ ). We are currently working on the application of two-lambda readout to array-multiplexed holograms.

This research was supported by the U.S. Air Force Office of Scientific Research under grant F4962092-J-0400. We thank Geoffrey Burr, Ernest Chuang, Allen Pu, Jean-Jacques Drolet, and Fai Mok for helpful discussions and Yayun Liu for technical assistance.

\section{References}

1. D. Psaltis, M. Levene, A. Pu, G. Barbastathis, and K. Curtis, Opt. Lett. 20, 782 (1995).

2. G. Barbastathis, M. Levene, and D. Psaltis, "Shift multiplexing with spherical reference waves," Appl. Opt. (to be published).

3. K. Wagner and D. Psaltis, Appl. Opt. 26, 5061 (1987).

4. H.-Y. S. Li and D. Psaltis, Appl. Opt. 33, 3764 (1994).

5. J. J. Amodei and D. L. Staebler, Appl. Phys. Lett. 18, 540 (1971).

6. F. Micheron and G. Bismuth, Appl. Phys. Lett. 20, 79 (1972).

7. Y. Qiao, S. Orlov, D. Psaltis, and R. R. Neurgaonkar, Opt. Lett. 18, 1004 (1993).

8. D. Brady, K. Hsu, and D. Psaltis, Opt. Lett. 15, 817 (1990).

9. D. Psaltis, F. Mok, and H.-Y. S. Li, Opt. Lett. 19, 210 (1994).

10. H. C. Külich, Opt. Commun. 64, 407 (1987).

11. J. D. Jackson, Classical Electrodynamics, 2nd ed. (Wiley, New York, 1975).

12. M. P. Petrov, S. I. Stepanov, and A. V. Khomenko, Photorefractive Crystals in Coherent Optical Systems (Springer-Verlag, Berlin, 1991). 